See Article page 578.

\section{Commentary: Do. Share. Discuss. Improve. Repeat.}

\section{Matthew L. Inra, MD, and Richard S. Lazzaro, MD}

In this issue of JTCVS Techniques, Sideris and Molena ${ }^{1}$ describe their robotic-assisted transthoracic repair of a giant left diaphragmatic hernia after the radical resection of metastatic ovarian cancer requiring bilateral diaphragm stripping. Their included video shows the important steps of the operation: port placement, reducing the contents of the hernia, lysis of adhesions from the diaphragm to abdominal viscera, partial primary closure of the defect, placement of intra-abdominal mesh and closure of the defect, and placement of intrathoracic mesh. The patient from this case report had an uneventful hospital stay and, at 6 months, had no recurrence of her hernia.

The case presented demonstrates the surgical technology available to thoracic surgeons today and how it translates into positive results for our patients. The da Vinci robot was originally created for telepresence surgery; open operations were performed with the robot in distant locations from the surgeon at the console. ${ }^{2}$ Now, the surgeon, the robot, and the patient are in the same operating room, allowing the robot to add to the armamentarium of the minimally invasive surgeon. Diaphragmatic hernia is a well-known surgical condition, and chronic hernias are generally approached through the chest. Without minimally invasive surgery, transthoracic access is achieved with a thoracotomy, leading to increased morbidity. ${ }^{3}$ Sideris and Molena ${ }^{1}$ preserved the surgical principles of the open operation through a minimally invasive approach. Thanks to the wristed robotic instruments, it was performed as if it were an open approach, as seen in their video. Because of their technique, their patient with metastatic cancer had a short, uncomplicated hospital stay and 6-month recovery,

From the Department of Cardiothoracic Surgery, Lenox Hill Hospital, New York, NY. Disclosures: The authors reported no conflicts of interest.

The Journal policy requires editors and reviewers to disclose conflicts of interest and to decline handling or reviewing manuscripts for which they may have a conflict of interest. The editors and reviewers of this article have no conflicts of interest.

Received for publication Aug 27, 2021; revisions received Aug 27, 2021; accepted for publication Sept 1, 2021; available ahead of print Sept 20, 2021.

Address for reprints: Richard S. Lazzaro, MD, Department of Cardiothoracic Surgery, Lenox Hill Hospital, 130 East 77th St, 4th Floor - Black Hall, New York, NY 10075 (E-mail: rlazzaro@northwell.edu).

JTCVS Techniques 2021;10:582-3

2666-2507

Copyright (c) 2021 The Author(s). Published by Elsevier Inc. on behalf of The American Association for Thoracic Surgery. This is an open access article under the CC BY-NC-ND license (http://creativecommons.org/licenses/by-nc-nd/4.0/).

https://doi.org/10.1016/j.xjtc.2021.09.014

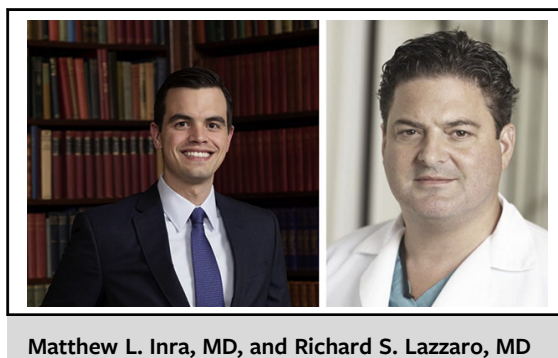

CENTRAL MESSAGE

Robotic surgery can allow surgeons to perform difficult operations and achieve excellent results. Sharing these successes and techniques will allow the field to review them and improve.

allowing her to receive systemic therapy as quickly as indicated, most importantly.

Another benefit of the robot is the video. ${ }^{1}$ All cases can be recorded. Video allows distribution and education. In some groups, video review and feedback lead to surgical improvement. ${ }^{4}$ Improvement leads to advancement and change. When complex operations are completed with the robot and that success is shared with the field, it inspires curiosity. We examine our own techniques: What is different about my repair? How can I improve my approach? If we can do this operation with the robot, what other difficult operations can we do? By honoring principles of robotic surgery like triangulation and port spacing, and preserving principles of a given operation, it is difficult to think what cannot be achieved by a robotic approach.

As video inspires curiosity, curiosity stirs debate. Sideris and Molena's technique involves 2 pieces of mesh in a "sandwich" fashion to reinforce the repair, despite being able to achieve a tension-free repair of the diaphragmatic defect without them. ${ }^{1}$ Again, we ask ourselves and others questions: Should we avoid mesh? It introduces a foreign body and exposes the patient to potential complications that could be avoided. Would certain patients benefit from mesh reinforcement? The diaphragm was stripped and might be weaker holding suture than diaphragm that was not. There is no literature supporting the fact that mesh definitively prevents recurrence, but in some cases it might; we do not know. This step in their technique will start conversation that could help lead us to a clearer answer. 
Sideris and Molena present a robotic technique for a well-described surgical condition with an excellent result. They tackle a difficult case with the robot, preserve the principles of the open operation, and show that it can be done well. The paper and video are educational and their use of mesh will spark a conversation. Let's keep using the robot, keep pushing the boundaries, and keep sharing our techniques. We, the patients and surgeons, will all benefit.

\section{References}

1. Sideris AC, Molena D. Robotic transthoracic diaphragmatic hernia repair. $J$ Thorac Cardiovasc Surg Tech. 2021;10:578-80.

2. Ballantyne GH, Moll F. The da Vinci telerobotic surgical system: the virtual operative field and telepresence surgery. Surg Clin North Am. 2003;83:1293-304.

3. Paul S, Nasar A, Port JL, Lee PC, Stiles BC, Nguyen AB, et al. Comparative analysis of diaphragmatic hernia repair outcomes using the nationwide inpatient sample database. Arch Surg. 2012;147:804.

4. Vaughn CJ, Kim E, O’Sullivan P, Huang E, Lin MY, Wyles S, et al. Peer video review and feedback improve performance in basic surgical skills. Am J Surg. 2016; 211:355-60. 